\title{
Direct Near-Field Optical Imaging of Higher Order Plasmonic Resonances
}

\author{
R. Esteban,,${ }^{\star \dagger}$ R. Vogelgesang,,,$\dagger$ J. Dorfmüller, ${ }^{\dagger}$ A. Dmitriev,,${ }^{\ddagger}$ C. Rockstuhl,,$\$$ \\ C. Etrich," and K. Kern ${ }^{\dagger, \perp}$
}

\begin{abstract}
Max Planck Institut für Festkörperforschung, 70569 Stuttgart, Germany, Bionanophotonics at Applied Physics, Chalmers University of Technology, 41296 Göteborg, Sweden, Institut für Festkörpertheorie and -optik and Institut für angewandte Physik, Friedrich-Schiller-Universität, 07743 Jena, Germany, and Institut de Physique des Nanostructures, Ecole Polytechnique Fédérale de Lausanne, CH-1015 Lausanne, Switzerland
\end{abstract}

Received May 15, 2008; Revised Manuscript Received July 17, 2008

\begin{abstract}
We map in real space and by purely optical means near-field optical information of localized surface plasmon polariton (LSPP) resonances excited in nanoscopic particles. We demonstrate that careful polarization control enables apertureless scanning near-field optical microscopy (aSNOM) to image dipolar and quadrupolar LSPPs of the bare sample with high fidelity in both amplitude and phase. This establishes a routine method for in situ optical microscopy of plasmonic and other resonant structures under ambient conditions.
\end{abstract}

When nanoscopic metallic structures are illuminated at adequate frequencies, the incoming radiation can couple to charge density oscillations and excite so-called localized surface plasmon polaritons (LSPPs). Recently, the nearfield enhancing qualities of LSPPs have been realized to hold promise for a bounty of novel applications in optics and photonics. ${ }^{1}$ These applications often rely on the fine details of LSPPs and their interactions with nanostructures. For example, in the fields of ultra sensitive bio(chemo)detectors ${ }^{2,3}$ or plasmonic metamaterials, ${ }^{4-6}$ direct near-field optical microscopy of LSPPs would be of great benefit. While spectroscopic far-field properties of LSPP resonances are routinely accessible, real-space near-field information is difficult to obtain.

To assess LSPPs of real nanostructures, microscopy techniques are required that are capable of spatially resolving the relevant structure sizes. One approach is the subsequent investigation by means of atomic force microscopy (AFM) of chemical or mechanical changes induced in suitable substrates by the excitation of optical eigenmodes. ${ }^{7,8} \mathrm{~A}$ second approach uses electron energy loss or induced cathode-luminescence scanning electron microscopy to map

* Corresponding authors. R.E., present address: Ecole Centrale Paris, Laboratoire em2c, CNRS, Châtenay-Malabry 92295 Cedex, France. Phone: +33 (1) 4113 1041. E-mail: ruben.esteban@em2c.ecp.fr. R.V., phone: +49 (711) 689 1581. Fax: +49 (711) 689 1662. E-mail: r.vogelgesang@fkf.mpg.de.

$\dagger$ Max Planck Institut für Festkörperforschung.

$\ddagger$ Chalmers University of Technology.

§ Institut für Festkörpertheorie and -optik, Friedrich-Schiller-Universität.

"Institut für angewandte Physik, Friedrich-Schiller-Universität.

${ }^{\perp}$ Ecole Polytechnique Fédérale de Lausanne.
LSPPs with nanometer resolution. ${ }^{9,10}$ This technique requires vacuum compatible samples.

For many applications in plasmonics, an all-optical detection of LSPPs with ultimate spatial resolution is called for. Characterizing local optical fields under ambient conditions has been achieved with AFM by carrying nanoscopic optical probes to the immediate vicinity of the nanostructures. ${ }^{11-16}$ However, such an optical probe has to perform contradicting tasks: as an "optical nano-antenna", its reception/emission efficiency improves with larger size, ${ }^{17}$ and as a near-field detector, the achievable spatial resolution improves with smaller size, which can also reduce parasitic interference and coupling effects between probe and sample. ${ }^{14}$ In this communication, we demonstrate that these competing demands can be concerted by polarization control of the exciting and scattered radiation, even with off-the-shelf AFM tips as optical probes. We are able to clearly map dipolar and quadrupolar LSPPs, both in phase and in amplitude.

In apertureless scanning near field optical microscopy (aSNOM), parasitic background signal from bulk scattering in the sample and tip is often suppressed by anharmonic lockin detection techniques, ${ }^{18,19}$ and homodyne, heterodyne, or pseudoheterodyne interferometry serves to amplify the signal and obtain phase information. ${ }^{20-23}$ However, the notorious interference and electromagnetic coupling of the probe tip especially to metallic samples continues to be a troublesome limiting factor. Often it prevents even qualitative interpretation of aSNOM images. A crucial aspect of our approach is therefore the orthogonal polarization of excitation and 


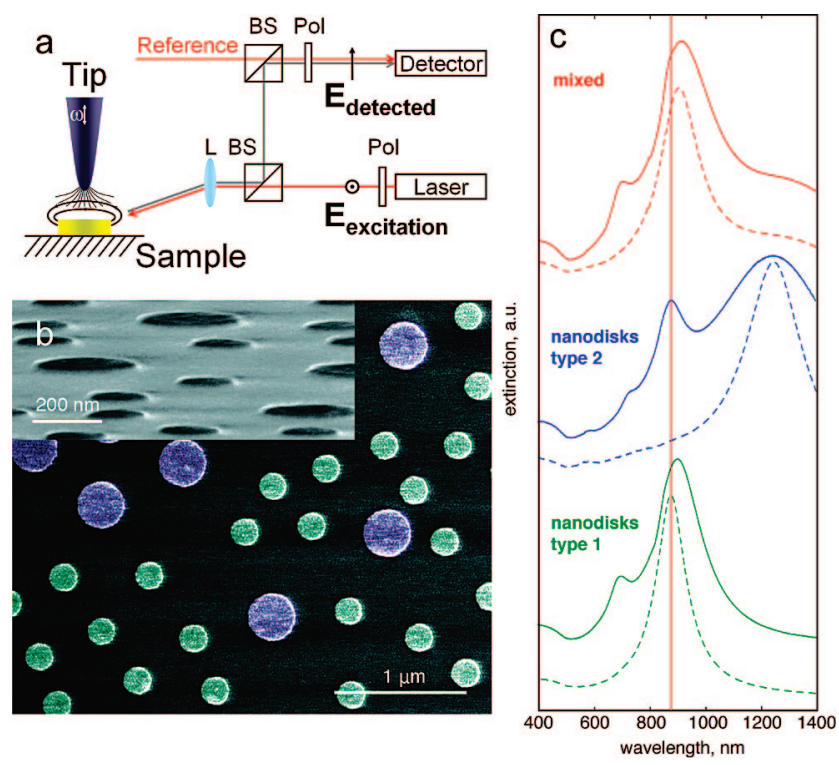

Figure 1. Near-field microscope setup, electron microscopy, and extinction spectroscopy of plasmonic structures. (a) aSNOM measuring scheme in cross-polarization configuration. Whereas the excitation beam is s-polarized, the p-polarized component of the scattered radiation is analyzed. Pol, polarizer; BS, beam splitter; L, lens. (b) Artificially colored SEM image illustrating the geometry of the investigated structures: mixed array of two nanodisk species with diameters $213 \mathrm{~nm}$ (highlighted in green) and $355 \mathrm{~nm}$ (highlighted in blue). Inset: a side view taken at $80^{\circ}$. (c) Extinction spectra collected with s-polarized excitation at normal incidence (dashed) and at $70^{\circ}$ off the substrate normal (solid) from arrays of nanodisks of diameters $213 \mathrm{~nm}$ (bottom, green), $355 \mathrm{~nm}$ (middle, blue), and the mixed array of 213 and $355 \mathrm{~nm}$ shown in (a) (top, red). The excitation line of the aSNOM setup $(875 \mathrm{~nm})$ is marked. Graphs are displaced vertically for clarity.

scattered radiation (Figure 1a.) As a first advantage, we note the substantial additional reduction of parasitic background signal. More notably, s-polarized radiation can be used to strongly excite the sample but not the probing AFM tip, which responds most strongly to fields parallel to the tip shaft, that is, normal to the sample surface. ${ }^{24-26}$

We illustrate the idea by expressing the Green propagator $\mathbf{G}=\mathbf{G}_{\mathbf{B}}+\mathbf{G}_{\mathbf{B}} \mathbf{T G}_{\mathbf{B}}$ of the full probe-sample system in a formal Born series in terms of the $\mathbf{T}$ matrices for isolated sample $\mathbf{T}^{(\mathrm{s})}$ and tip $\mathbf{T}^{(\mathrm{t})}$,

$$
\begin{aligned}
\mathbf{T}= & \mathbf{T}^{(\mathrm{t})}+\mathbf{T}^{(\mathrm{s})} \cdot \mathbf{T}^{(\mathrm{t})}+\mathbf{T}^{(\mathrm{t})} \cdot \mathbf{T}^{(\mathrm{s})} \cdot \mathbf{T}^{(\mathrm{t})}+\ldots \\
& +\mathbf{T}^{(\mathrm{s})}+\mathbf{T}^{(\mathrm{t})} \cdot \mathbf{T}^{(\mathrm{s})}+\mathbf{T}^{(\mathrm{s})} \cdot \mathbf{T}^{(\mathrm{t})} \cdot \mathbf{T}^{(\mathrm{s})}+\ldots
\end{aligned}
$$

"•" represents the Green propagator $\mathbf{G}_{\mathbf{B}}$ in the background medium. An external excitation field $\mathbf{E}^{(\mathrm{exc})}$ leads to the field $\mathbf{E}^{\text {(sca) }}=\mathbf{G}_{\mathbf{B}} \mathbf{T E}^{(\mathrm{exc})}$ being scattered toward the detector. Through a periodic oscillation of the probe tip above the sample and subsequent Fourier filtering of the signal at a harmonic of the modulation frequency $\omega$, the stationary sample contribution $\mathbf{G}_{\mathbf{B}} \mathbf{T}^{(\mathrm{s})} \mathbf{E}^{(\mathrm{exc})}$ to the scattered radiation is eliminated. The s-polarized excitation is considered for its electric field vector parallel to the sample surface and perpendicular to the shaft of our probing AFM tip (Figure 1a.) The backscattered radiation is interferometrically analyzed such that the p-component is selected. This reduces strongly the terms $\mathbf{G}_{\mathbf{B}}\left[\mathbf{T}^{(\mathrm{t})}+\mathbf{T}^{(\mathrm{s})} \cdot \mathbf{T}^{(\mathrm{t})}+\mathbf{T}^{(\mathrm{t} \bullet} \cdot \mathbf{T}^{(\mathrm{s})} \cdot \mathbf{T}^{(\mathrm{t})}+\ldots\right] \mathbf{E}^{(\mathrm{exc})}$ with $\mathbf{T}^{(t)}$ as the leading factor and leaves terms with leading
$\mathbf{T}^{(\mathrm{s})}$, whose off-diagonal elements are related with polarization rotation effects of the sample. To lowest order, the Born series thus reads

$$
\mathbf{T} \approx \mathbf{T}^{(\mathrm{t})} \bullet \mathbf{T}^{(\mathrm{s})}=\mathbf{T}^{(\mathrm{t})} \mathbf{G}_{\mathrm{B}} \mathbf{T}^{(\mathrm{s})}
$$

Higher order contributions with leading factor $\mathbf{T}^{(\mathrm{s})}$, which describe undesired coupling effects between sample and probe tip, diminish rapidly with the order unless the tip-sample system exhibits joint resonances. In the present study and probably more generally, such coupling terms may be neglected as corroborated by the excellent agreement of experimentally recorded near-field images of LSPPs and the numerical simulations that were undertaken without considering the presence of any local probe.

Interpreting eq 2, we may describe the scattering process

$$
\mathbf{E}^{(\text {sca })} \approx \mathbf{G}_{\mathrm{B}} \mathbf{T}^{(\mathrm{t})} \mathbf{G}_{\mathrm{B}} \mathbf{T}^{(\mathrm{s})} \mathbf{E}^{(\mathrm{exc})}
$$

in cross-polarization aSNOM as a linear chain of action and reaction: s-polarized radiation excites the sample only. The induced localized sample fields strongly excite the tip wherever they exhibit field components along the tip shaft because only they can match the (near) rotational symmetry of the strongest tip response at the apex, ${ }^{17,25}$ whereas orthogonal sample field components are symmetry mismatched. Thus, the dominant tip mode communicates the local electric field component parallel to the tip ${ }^{17,22,27,28}$ by scattering radiation toward the detector, which is detected as p-polarized light.

Using the advantages of the described experimental scheme, we image dipolar and quadrupolar LSPPs of Au nanodisks, fabricated on glass substrates by hole-mask colloidal lithography (Figure 1b,c and ref 29). In brief, the nanofabrication method uses self-assembly of charged colloidal beads on the surface of poly(methyl methacrylate) (PMMA), spin-coated on the supporting sample surface. Evaporation of thin metal film on top of short-range ordered beads and their subsequent removal by tape-stripping followed by the oxygen plasma etch creates the evaporation mask composed of thin metal film with nanoscopic holes, arranged in the short-range macroscopic pattern. For the purpose of the present work, it was possible to obtain several species of nanodisks on the same substrate, in our case, of diameters 213 and $355 \mathrm{~nm}$ by employing the mixture of nominal polystyrene (PS) particle diameters of 170 and 300 $\mathrm{nm}$. Resulting arrays of $20 \mathrm{~nm}$ high nanodisks have a narrow size distribution of about $4 \%$ standard deviation with nearestneighbor surface-to-surface distances between nanodisks on the order of the smallest diameter in the mixture.

In the far-field, we characterize the samples by extinction spectroscopy with s-polarized radiation incident at different angles (Varian Cary 500, illumination area: $5 \times 10 \mathrm{~mm}$ ). Despite some ensemble averaging effects such as inhomogeneous broadening due to the finite size distribution of the nanodisks in the macroscopic array, the spectra in Figure 1c are representative of the spectra of individual nanoparticles, assumed to be noninteracting in the array. ${ }^{30}$ Under normal incidence, the small and large nanodisk species exhibit distinct extinction peaks; at wavelengths $\sim 880$ and $\sim 1250$ $\mathrm{nm}$, respectively. By tilting the angle of incidence to $70^{\circ}$, we find this extinction peak evolves only qualitatively. More 


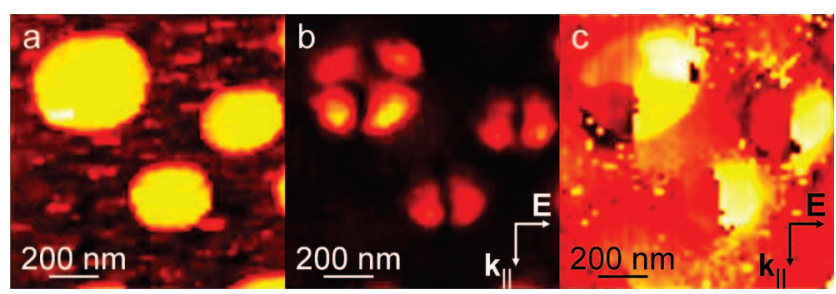

Figure 2. Recorded near field optical image of a $1.1 \times 1.1 \mu \mathrm{m}^{2}$ area containing only one large and two small nanodisks. (a) Topography, (b) optical amplitude, and (c) optical phase. Excitation of wavelength $875 \mathrm{~nm}$ and second harmonic demodulation were used. The directions of the electric field vector and of the projection of the excitation wavevector onto the substrate are indicated.

striking is the concurrent emergence of a second peak on the high-energy shoulders at wavelengths $\sim 695 \mathrm{~nm}$ and $\sim 880 \mathrm{~nm}$, respectively. We note that the low-energy extinction resonance of the small nanodisks spectrally coincides with the high-energy resonance of the large nanodisks. The extinction spectrum of a mixed array, in which both 213 and $355 \mathrm{~nm}$ diameter species are present in a proportion of $\sim 6$ : 1 , is consistent with a superposition of the individual resonances.

Led by physical intuition, we can attribute the low-energy spectral signatures to dipolar LSPPs in the nanodisks. Because of symmetry selection rules, these may be excited by linearly polarized light under normal incidence but no resonant quadrupolar response is expected. Quadrupoles become accessible under oblique incidence and s-polarization, when field polarization breaks the symmetry between "left" and "right" and retardation between "front" and "back". To test these interpretations in the near-field, we investigate with our aSNOM approach (Figure 1a and refs 21 and 31) arrays containing both nanodisk species on a single substrate with s-polarized excitation of wavelength $\sim 875 \mathrm{~nm}$ (marked in Figure 1c).

The incoming radiation is focused close to the apex of a standard, noncontact silicon AFM tip (Nanosensors AdvancedTEC NC) without any metallic coating, mounted on a Park Scientific M-5 AFM. The sample is scanned horizontally while the stationary tip is operated at constant distance mode with an oscillation amplitude on the order of $\sim 20 \mathrm{~nm}$. The AFM deflection signal is directly used to generate a synchronized second harmonic reference signal for the lock-in demodulation of the photoelectric signal. Before the p-component of the back-scattered radiation is selected by a polarizer, it is amplified interferometrically with a reference beam. The photoelectric signal is therefore not proportional to scattered intensity but the electric field itself. ${ }^{20-23}$ Recording it twice with a relative phase shift of $90^{\circ}$ in the reference yields optical amplitude and phase information.

Figure 2 (and Figure 4) show representative measurement results for small and large nanodisks. As anticipated, a strong correlation between nanodisk size and near-field signature is found. The small nanodisks show two lobes of strong amplitude and a phase jump close to $180^{\circ}$, that is, a sign change between the lobes, characteristic of dipolar resonances. The nodal line is approximately perpendicular to the
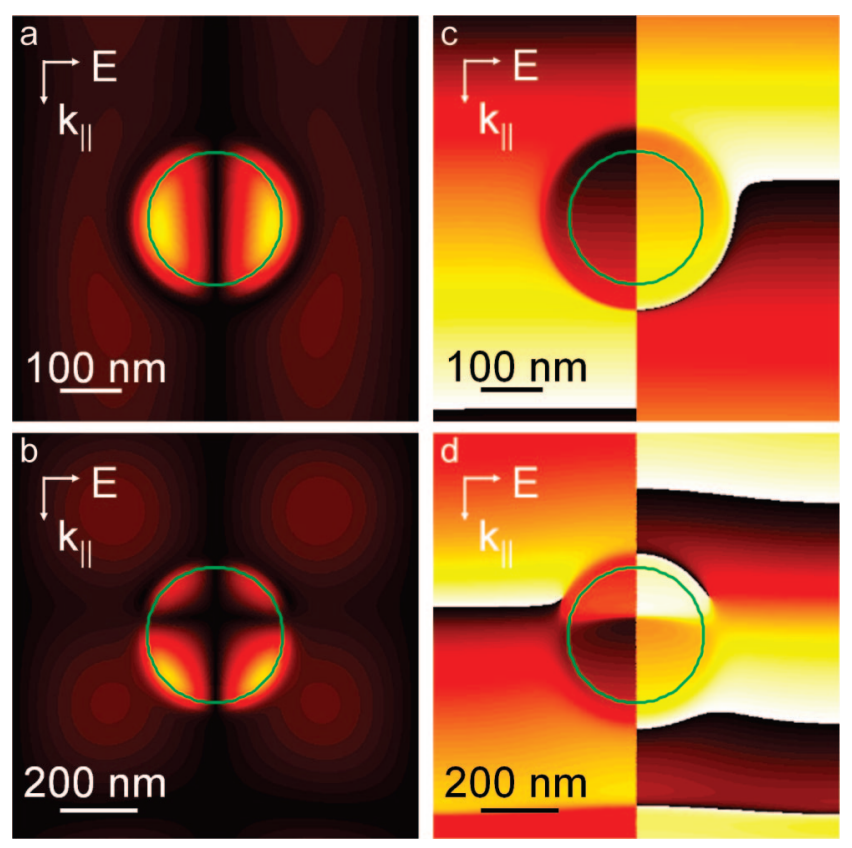

Figure 3. Simulated near-field images. Amplitude (a) and phase (c) of the recorded signal; calculated for a wavelength of $875 \mathrm{~nm}$ for nanodisks of $216 \mathrm{~nm}$ diameter and $\sim 20 \mathrm{~nm}$ thickness. The phase is plotted between -180 and $180^{\circ}$. (b,d) equivalent results for nanodisks of $350 \mathrm{~nm}$ diameter. Circles indicate the rim of the nanodisks, and the directions of the electric vector and the projection of the excitation wavevector onto the substrate are also shown. The spatial discretisation is $\Delta x=2 \mathrm{~nm}$, the temporal $\Delta x / 2 c$.

direction of the exciting electric field. The signal from the larger nanodisk exhibits a quadrupolar pattern, with four amplitude lobes and corresponding areas of distinct phase values. Here, both the electric field and the projection of the propagating vector onto the substrate define the symmetries of the observed lobes.

For comparison, we perform finite-difference time-domain simulations $^{32}$ of corresponding nanodisks on a substrate. The geometry and the excitation conditions were chosen as in the experiments, namely, an s-polarized plane wave illuminates the structure at an incidence angle of $70^{\circ}$. The polarizability of gold is simulated assuming a Drude model. In the Drude model, the free parameters are selected to match the permittivity of gold $(\epsilon=-30.5491+1.8539 i)$ at the operating wavelength $(\lambda=875 \mathrm{~nm})$. Glass is assumed as the substrate with a permittivity of 2.25 . In the simulations, the nanodisks are arranged periodically; periods of 660 and $1050 \mathrm{~nm}$ are assumed for the small (Figure 3a) and large (Figure 3b) nanodisk, respectively. We have verified that changing the period or simulating isolated nanodisks does not notably change the results. In the $z$ direction (normal to the substrate surface), the computational space is truncated with perfectly matched layers. In the $x$ and in the $y$ direction, Bloch periodic boundaries are used. The probing AFM tip and the demodulation are not included in the simulation. Instead, we construct the detected signal as produced by a point-like nanoantenna with a highly anisotropic dipolepolarizability ${ }^{27}$ that probes the electric field (normal to the surface) at an effective average distance of $30 \mathrm{~nm}$ to the sample, and then re-emits p-polarized radiation toward 

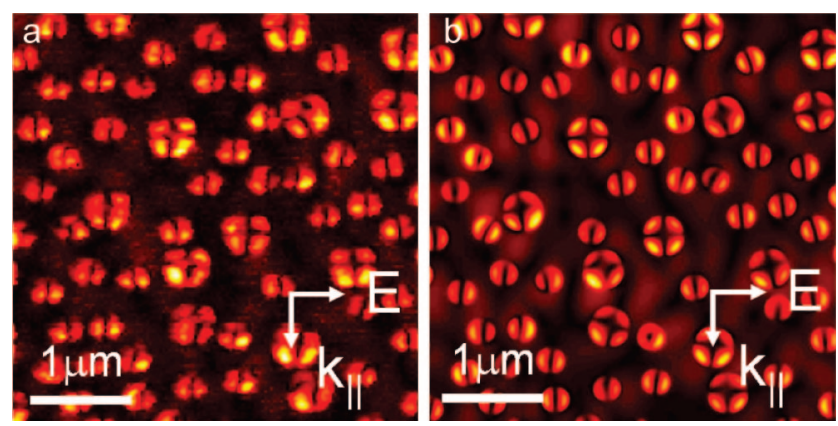

Figure 4. (a) Measured optical near-field amplitude for a $4 \times 4$ $\mu \mathrm{m}^{2}$ containing some 70 nanodisks, including those in Figure 2. The excitation wavelength is $875 \mathrm{~nm}$ and second harmonic demodulation was used. (b) Simulation of the measured situation of (a). The diameter of the large nanodisks is $350 \mathrm{~nm}$ and for the small disks $216 \mathrm{~nm}$. The periodicity here was $5 \mu \mathrm{m}$, the spatial discretisation $\Delta x=4 \mathrm{~nm}$ and the temporal $\Delta x / 2 c$. The directions of the electric field vector and of the projection of the excitation wavevector onto the substrate are indicated.

the detector. The striking resemblance of experimentally recorded (Figure 2) and simulated near-field images (Figure 3) both in amplitude and phase justifies our modeling assumptions.

The discussion up to now has implicitly assumed that the main features of the resonances of each individual particle are not noticeably affected by the presence of neighboring structures. The interparticle separation is large enough to avoid the strong near-field interaction that can lead to coupled resonances. Because of the absence of long-range order, grating effects are not present in our experimental samples, either. Still, scattering from the varying arrangements of nearest neighbor nanodisks may alter the total excitation field at each nanodisk in a slightly different manner. While we expect the main features of the resonant modes to remain the same, it is interesting to study the influence of the local environment on the near field behavior of resonant structures.

Figure 4a shows the measured amplitude over a representative larger area containing some 70 nanodisks. For comparison, we show in Figure $4 \mathrm{~b}$ a simulation corresponding to Figure 4a. We observe that essentially all individual nanodisks are excited and respond with clear near-field patterns. Generally, they are still classifiable as dipolar or quadrupolar resonances for small or large nanodisks, respectively, but the individual field distributions do exhibit small variations from nanodisk to nanodisk. Notably, the strength of the resonance and the orientation of the symmetry axis in the sample plane are not identical for all nanodisks. Occasionally, equivalent lobes on the same individual nanodisk do not appear as symmetric as Figure 3 would suggest for isolated nanodisks. Finally, in quadrupolar mode patterns, a certain trend of lobe confluence can be made out along the $k_{\|}$direction. Nonetheless, all of these effects are reproduced within the simulations in considerable detail, in spite of the drastic assumption of considering the vertical field component of bare samples without any local probe. We take these observations as evidence that our cross-polarization scheme decouples sample and probe tip in a very effective manner, thus supplying easy to interpret, undisturbed microscopic information of higher order plasmonic resonance modes with subwavelength resolution.

To summarize, we have demonstrated in this communication how the combination of aSNOM with a cross polarization scheme results in a reliable method to image localized surface plasmonic resonances beyond dipolar modes with spatial detail well-below relevant structure sizes and with much reduced background signal. We achieve simultaneous mapping of dipolar and quadrupolar LSPPs for Au nanodisks of different diameter. The excellent agreement between interpretations of extinction spectroscopy, near-field optical microscopy, and theoretical simulations affirms the approach. Wherever spurious signals due to parasitic sample-probe coupling are problematic, cross-polarized aSNOM may facilitate unperturbed information. It opens the door to detailed in situ studies of individual resonant nanostructures, in basic science as well as assessment of design and fabrication parameters. Our approach works under ambient conditions and is applicable not only to LSPPs and the aforementioned plasmonic detectors and meta-materials but also to a large variety of near-field optical phenomena, such as optical nanoantennae, dielectric microresonators, subwavelength apertures, or propagating plasmon polaritons at appropriately shaped one- and two-dimensional metal structures.

\section{References}

(1) Lal, S.; Link, S.; Halas, N. J. Nature Photonics 2007, 1, 641-648.

(2) Aizpurua, J.; Hanarp, P.; Sutherland, D. S.; Kall, M.; Bryant, G. W.; de Abajo, F. J. G. Phys. Rev. Lett. 2003, 90, 057401.

(3) Hao, F.; Nehl, C. L.; Hafner, J. H.; Nordlander, P. Nano Lett. 2007, 7, 729-732.

(4) Rockstuhl, C.; Zentgraf, T.; Guo, H.; Liu, N.; Etrich, C.; Loa, I.; Syassen, K.; Kuhl, J.; Lederer, F.; Giessen, H. Appl. Phys. B: Lasers And Optics 2006, 84, 219-227.

(5) Shalaev, V. M. Nature Photonics 2007, 1, 41-48.

(6) Dmitriev, A.; Pakizeh, T.; Käll, M.; Sutherland, D. S. Small 2007, 3, 294-299.

(7) Bachelot, R.; H’Dhili, F.; Barchiesi, D.; Lerondel, G.; Fikri, R.; Royer, P.; Landraud, N.; Peretti, J.; Chaput, F.; Larnpel, G.; Boilot, J. P.; Lahlil, K. J. Appl. Phys. 2003, 94, 2060-2072.

(8) Leiderer, P.; Bartels, C.; Konig-Birk, J.; Mosbacher, M.; Boneberg, J. Appl. Phys. Lett. 2004, 85, 5370-5372.

(9) Nelayah, J.; Kociak, M.; Stephan, O.; de Abajo, F. J. G.; Tence, M.; Henrard, L.; Taverna, D.; Pastoriza-Santos, I.; Liz-Marzan, L. M.; Colliex, C. Nat. Phys. 2007, 3, 348-353.

(10) Vesseur, E. J. R.; de Waele, R.; Kuttge, M.; Polman, A. Nano Lett. 2007, 7, 2843-2846.

(11) Zenhausern, F.; Martin, Y.; Wickramasinghe, H. K. Science 1995, 269, 1083-1085.

(12) Knoll, B.; Keilmann, F. Nature 1999, 399, 134-137.

(13) Hillenbrand, R.; Keilmann, F. Appl. Phys. B: Laser Opt. 2001, 73, 239-243.

(14) Hillenbrand, R.; Keilmann, F.; Hanarp, P.; Sutherland, D. S.; Aizpurua, J. Appl. Phys. Lett. 2003, 83, 368.

(15) Anderson, N.; Bouhelier, A.; Novotny, L. J. Opt. A: Pure. Appl. Opt. 2006, 8, 227-233.

(16) Lee, K. G.; Kihm, H. W.; Kihm, J. E.; Choi, W. J.; Kim, H.; Ropers, C.; Park, D. J.; Yoon, Y. C.; Choi, S. B.; Woo, H.; Kim, J.; Lee, B.; Park, Q. H.; Lienau, C.; Kim, D. S. Nature Photonics 2007, 1, 5356.

(17) Esteban, R.; Vogelgesang, R.; Kern, K. Nanotechnology 2006, 17, 475-482.

(18) Hillenbrand, R.; Knoll, B.; Keilmann, F. J. Microscopy 2001, 202, 77.

(19) Walford, J. N.; Porto, J. A.; Carminati, R.; Greffet, J.-J.; Adam, P. M.; Hudlet, S.; Bijeon, J.-L.; Stashkevich, A.; Royer, P. J. Appl. Phys. 2001, 89, 5159-5169.

(20) Hillenbrand, R.; Keilmann, F. Phys. Rev. Lett. 2000, 85, 3029-3032. 
(21) Bek, A.; Vogelgesang, R.; Kern, K. Rev. Sci. Instrum. 2006, 77, 043703.

(22) Gomez, L.; Bachelot, R.; Bouhelier, A.; Wiederrecht, G. P.; Chang, S.-H.; K, G. S.; Hua, F.; Jeon, S.; Rogers, J. A.; Castro, M. E.; Blaize, S.; Stefanon, I.; Lereondel, G.; Royer, P. J. Opt. Soc. Am. B 2006, 23, 823-833.

(23) Ocelic, N.; Huber, A.; Hillenbrand, R. App. Phys. Lett. 2006, 89, 101124.

(24) Martin, O. J. F.; Girard, C. Appl. Phys. Lett. 1997, 70, 705-707.

(25) Novotny, L.; Bian, R. X.; Xie, X. S. Phys. Rev. Lett. 1997, 79, 645648.

(26) Micic, M.; Klymyshyn, N.; Suh, Y. D.; Lu, H. P. J. Phys. Chem. B 2003, 107, 1574-1584.

(27) Bouhelier, A.; Beversluis, M.; Hartschuh, A.; Novotny, L. Phys. Rev. Lett. 2003, 90, 013903.
(28) Thomas, M.; Greffet, J. J.; Carminati, R.; Arias-Gonzalez, J. R. Appl. Phys. Lett. 2004, 85, 3863-3865.

(29) Fredriksson, H.; Alaverdyan, Y.; Dmitriev, A.; Langhammer, C.; Sutherland, D. S.; Zäch, M.; Kasemo, B. Adv. Mater. 2007, 19, 42974302.

(30) Hanarp, P.; Kall, M.; Sutherland, D. S. J. Phys. Chem. B 2003, 107, 5768-5772.

(31) Esteban, R. Ph D. thesis, Ecole Polytechnique Fédérale de Lausanne, 2007.

(32) Taflove, A.; Hagness, S. C. Computational Electrodynamics: The Finite-Difference Time-Domain Method, 3rd ed.; Artech House, 2005.

NL801396R 